\title{
PHOTOINDUCED PHENOMENA IN SULPHUR CRYSTALS AND FILMS
}

\author{
Ya.O. DovgII, I.V. KitYK and O.G. YablonovsKa \\ Experimental Physics Department, Lviv University \\ Yaroslavenko 47/31, 290034 Lviv-34, Ukraine
}

(Received September 2, 1991; revised version December 11, 1991)

The irradiation of sulphur crystals and films with ultraviolet light induces the change of transmission in the visible part of spectra. It was found that these phenomena exist only at low temperatures $(4.2-50 \mathrm{~K})$. The behaviour of optical transparency as a function of time after switching the illumination off was investigated. These dependences are essentially different for the films and crystals. In order to understand the origin of these phenomena the structural investigation was conducted. Very unusual behaviour of darkening kinetics was obtained.

PACS numbers: 72.40.+w, 71.55.Gs, 78.50.Ge

In the recent years the interest in nonsilver photographic compounds has grown rapidly due to the high expense of silver. But on today no compounds have been found which would posess the high contrast in the low temperature range.

Experimental investigations of the photoinduced properties of phosphorus and arsenicum crystals have also been reported [1-3]. The additional absorption under the ultraviolet light in crystal $\mathbf{P}$ and As is caused by the shift of energy gap. The analogous measurements on the sulphur crystals were conducted by authors in $[4,5]$ and in $[6]$ with the use of X-rays. Despite the effort, very little is known about the mechanism of the photoinduced changes in the nonsilver halides [7-10].

In this report we present an experimental investigation of the photoinduced changes caused by UV-irradiation in sulphur crystals and films. The crystals belong to the space group $D_{2 h}^{24}$. The samples were prepared from natural minerals of different quality. The quality was checked by the number of dislocations. The size of crystal surface was $12 \times 12 \mathrm{~mm}^{2}$ and thickness near $5 \mathrm{~mm}$. The films were evaporated on the quartz surfaces in vacuum near $10^{-7}$ torr. The samples were mounted in the helium cryostat made in the Institute of Ukrainian Physics. They were irradiated using focused UV-light with $\lambda \approx 331 \mathrm{~nm}$. The transmission and reflection spectra of samples were recorded using the Specord-M40 spectrophotometer. The photostructural changes and concentration of dislocations were registered using an $\mathrm{X}$-ray diffractometer. 

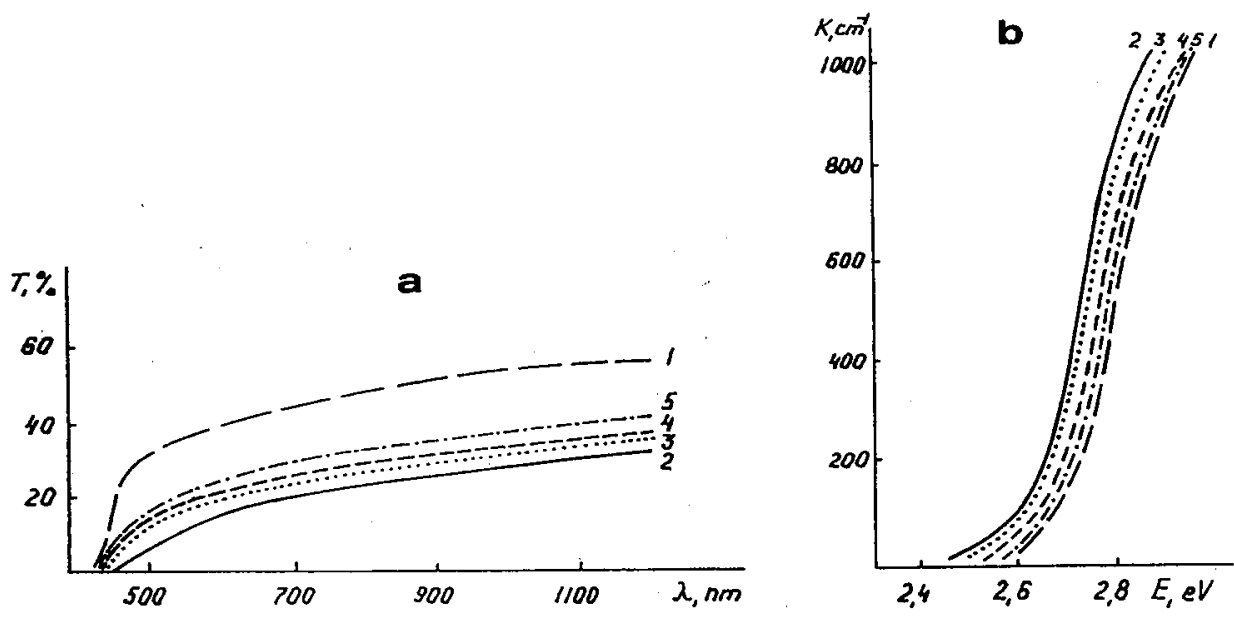

Fig. 1. (a) Transmission of $\alpha$-S crystal: 1 - before the irradiation, $2-18$ hours, $3-24$ hours, $4-120$ hours, $5-1000$ hours after switching the irradiation off. (b) The same for the absorption spectra $\left(T=4.5 \mathrm{~K}, \lambda_{0}=331 \mathrm{~nm}\right.$, duration of irradiation $\tau_{0}=30 \mathrm{~min}$ ).

The transmission $T(\lambda)$ spectra of the irradiated crystals from the time after switching the irradiation off are plotted in Fig. 1a. The illumination was conducted at the liquid helium temperature $\left(T_{c}=4.5 \mathrm{~K}\right)$ with light of $331 \mathrm{~nm}$ wavelength. Then the crystals were kept in the darkness at room temperature. At the beginning after the 30-minute irradiation changes of the crystal colour were not essentially found. The colouration process developed then in the following way. After 10-15 hours of keeping of the crystals in the darkness on the surface of the crystals the changes of transparency and colours (the browning) were clearly seen. This process reached its maxima at approximately 22 hours after switching the illumination off (the second and third curves in Fig. 1). The reversible processes occurred very slowly. For example, the third curve shows the decrease of the transmission 24 hours after switching the irradiation off and the fifth curve - the reversible increase 1000 hours after. Such long relaxation times can mean the great role of submolecular aggregates, which are reoriented by the external optical perturbation due to destroying of the chemical bonds within the $S_{8}$ molecule. Reflection spectra $R(\lambda)$ near the band edge were measured simultaneously. Using the expression (1) the absorption coefficients were calculated and they are plotted in Fig. 1b:

$$
K=\frac{1}{d} \ln \left\{\frac{1-R}{2 T}+\left[\frac{(1-R)^{2}}{4 T^{2}}+4 R^{2}\right]^{1 / 2}\right\} .
$$

It is necessary to note the existence of photoinduced changes in reflection coefficients, too.

Using the hydrogen light sources without the filters tends to the similar effect. At room temperature there were not any photostimulated changes. Therefore the 
changes of transparency were increased with the decrease of temperature to $77 \mathrm{~K}$. Disappearance of the browning took place with the increase of temperature up to $365 \mathrm{~K}$. It is very interesting to notice that the change of transparency essentially depends on the concentration of dislocations. The increase of dislocation numbers tends to the decrease of corresponding phenomena.

The change of colour which appeared after the radiation was observed only in the vicinity of sample surface $(\sim 1 \mu \mathrm{m})$. The little depth of this layer complicated its investigations. The additional absorption arose from the near-surface layer.

For the more detailed investigation of the origin of this feature we carried out the analogous study on the sulphur films. For obtaining information about the structural changes we carried out $\mathrm{X}$-ray diffraction measurements. Experimental investigations showed that the sulphur film consists of two parts: rhombical crystalline and amorphous substance. The irradiation tends to the shift of the main structural reflexes in $\alpha$-crystalline phase on $8-10^{\prime}$ with the accuracy of the reflexe position determination near $2^{\prime}$.

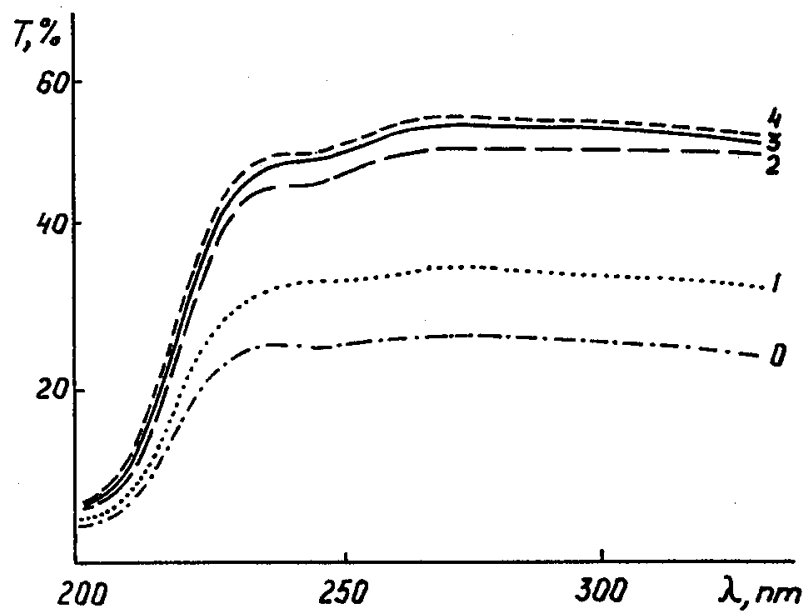

Fig. 2. Transmission spectra $(1,2)\left(\right.$ at $\left.T=4.5 \mathrm{~K}, \lambda_{0}=331 \mathrm{~nm}\right)$ of irradiated film of sulphur and nonilluminated one (keeping at $\left.T=4.5 \mathrm{~K}, \tau_{0}=15 \mathrm{~min}\right)(3,4),(1,3)-$ $40 \mathrm{~min},(2,4)-20$ hours after switching the illumination off, $0-$ before the irradiation.

The detailed microscopical investigations of the films indicated the occurring of inhomogeneous surface under the UV-light radiation. Due to the thin thickness of the films the transparence edge is shifted to the short-wavelength range (Fig. 2). The observed changes are caused probably by distorted submolecular aggregates created by light at helium temperature. The curves $1,2,3,4$, also as in the case of crystals, go parallel one to another. The darkening rose in the first 30 minutes after illumination.

From the conducted experimental and theoretical investigations one can propose the following origin of phenomena. The irradiation of samples with the light of $331 \mathrm{~nm}$ wavelength (the quantum energy $\approx 3.8 \mathrm{eV}$ ) tends to destroying of 
the chemical bonds within the $\mathrm{S}_{8}$ molecule (the minimal energy of bond within the molecule is equal near $3.5 \mathrm{eV}$ ) and to reorientation of submolecular aggregates. The main role in the pecularities of the kinetics of colouration was played by the local defects which create the metastable states within the band energy gap. Due to the electron-phonon interaction the energy of these levels was shifted what tends to the additional absorption and change of colours. Such assumption was confirmed by the independent measurement of infrared transmission spectra immediately after illumination [11].

The absorption coefficient of the $\alpha$-S crystals (at $4.5 \mathrm{~K}$ ) for $\lambda=331 \mathrm{~nm}$ is about $5 \times 10^{3} \mathrm{~cm}^{-1}$. The corresponding value at the room temperature is about $7 \times 10^{4} \mathrm{~cm}^{-1}[12,13]$. In the first case the UV-light $(331 \mathrm{~nm})$ penetrates the $\alpha-\mathrm{S}$ sample about $2 \mu \mathrm{m}$ deep. The layer with the described change of colour is just of the same thickness. In the second case the penetration depth is about $0.12 \mu \mathrm{m}$ and therefore the corresponding effect is very small.

\section{References}

[1] A.N. Rodionov, R.I. Kalendarev, A.V. Shendrik, Yu.R. Zakis, Phys. Status Solidi A 79, K151 (1983).

[2] R.I. Kalendarev, A.I. Sazanov, A.N. Rodionov, G.V. Chikvaidze, J.A. Eiduss, Mater. Res. Bull. 19, 11 (1983).

[3] A.I. Sazanov, R.I. Kalendarev, Izv. Akad. Nauk Latv. SSR Ser. Phys. Tekhn. 3, 35 (1983).

[4] Ya.O. Dovgii, I.V. Kityk, O.G. Yablonovska, Pisma Zh. Tekh. Fiz. 15, No 15, 69 (1989) (in Russian).

[5] Ya.O. Dovgii, I.V. Kityk, O.G. Yablonovska, Ukr. Fiz. Zh. 35, No 6, 908 (1990) (in Ukrainian).

[6] R.M. Nelson, W.D. Smythe, B.W. Hapke, A.J. Cohen, Icarus 85, No 2, 326 (1990):

[7] K.K. Schwartz, The Physics of Optical Recording in Dielectrics and Semiconductors, Zinatne, Riga 1986.

[8] I.V. Kityk, Zh. Prikl. Spektrosk. 42, 487 (1985).

[9] N.F. Mott, E.A. Davis, Philos. Mag. A 32, 961 (1987).

[10] M.L. Klinger, V.G. Karpov, JETP 82, 1687 (1982).

[11] Ya.O. Dovgii, I.V. Kityk, O.G. Yablonovska, unpublished data.

[12] W.E. Spear, A.R. Adams, J. Phys. Chem. Sol. 27, 281 (1966).

[13] A.K. Abas, N.H. Ahmad, J. Phys. Chem. Sol. 47, No 2, 143 (1986). 